\title{
Normal structure of Lorentz-Orlicz spaces
}

\author{
by Pei-Kee Lin (Memphis, Tenn.) and Huiying Sun (Harbin)
}

Abstract. Let $\phi: \mathbb{R} \rightarrow \mathbb{R}_{+} \cup\{0\}$ be an even convex continuous function with $\phi(0)=0$ and $\phi(u)>0$ for all $u>0$ and let $w$ be a weight function. $u_{0}$ and $v_{0}$ are defined by

$$
u_{0}=\sup \{u: \phi \text { is linear on }(0, u)\}, \quad v_{0}=\sup \{v: w \text { is constant on }(0, v)\}
$$

(where $\sup \emptyset=0$ ). We prove the following theorem.

Theorem. Suppose that $\Lambda_{\phi, w}(0, \infty)$ (respectively, $\left.\Lambda_{\phi, w}(0,1)\right)$ is an order continuous Lorentz-Orlicz space.

(1) $\Lambda_{\phi, w}$ has normal structure if and only if $u_{0}=0$ (respectively, $\int_{0}^{v_{0}} \phi\left(u_{0}\right) \cdot w<2$ and $\left.u_{0}<\infty\right)$.

(2) $\Lambda_{\phi, w}$ has weakly normal structure if and only if $\int_{0}^{v_{0}} \phi\left(u_{0}\right) \cdot w<2$.

1. Introduction. Let $\Omega$ denote either $[0,1]$ or $[0, \infty)$ and $m$ denote the Lebesgue measure on $\Omega$. For a measurable function $x$ on $\Omega$, the distribution function $d_{x}$ and the decreasing rearrangement $x^{*}$ are defined by

$$
d_{x}(t)=m(|x|>t), \quad x^{*}(t)=\inf \left\{s>0: d_{x}(s) \leq t\right\} .
$$

An even convex continuous function $\phi: \mathbb{R} \rightarrow \mathbb{R}_{+} \cup\{0\}$ is said to be a Young function if $\phi(0)=0$ and $\phi(u)>0$ for all $u \neq 0$. A function $w: \Omega \rightarrow \mathbb{R}_{+}$is called a weight function if it is a nonincreasing left continuous function and

$$
\int_{0}^{1} w(t) d t=1 .
$$

For a Young function $\phi$ and a weight function $w$, the associated LorentzOrlicz space $\Lambda_{\phi, w}(\Omega)$ (or $\Lambda_{\phi, w}$ for short) is the set of all real measurable functions $x$ on $\Omega$ such that

$$
\varrho_{\phi}(\lambda x)=\int_{\Omega} \phi\left(\lambda x^{*}(t)\right) w(t) d t \equiv \int_{\Omega} \phi\left(\lambda x^{*}\right) w<\infty
$$

1991 Mathematics Subject Classification: 46B20, 46B42.

Key words and phrases: Lorentz-Orlicz space, normal sturcture, order continuous, Young function. 
for some $\lambda>0$. The norm of $x \in \Lambda_{\phi, w}$ is defined by

$$
\|x\|=\inf \left\{\varepsilon>0: \varrho_{\phi}(x / \varepsilon) \leq 1\right\} .
$$

Recall that a mapping $\sigma: \mathbb{R}_{+} \rightarrow \mathbb{R}_{+}$is said to be a measure preserving transformation if for any measurable set $D, m(D)=m\left(\sigma^{-1}(D)\right)$. It is known that for any measure preserving transformation $\sigma$ and any $x \in \Lambda_{\phi, w}, x^{*}=$ $(x \circ \sigma)^{*}$ and

$$
\int \phi\left(x^{*}\right) w \geq \int \phi(x) w \circ \sigma .
$$

It is also known that for $z \in \Lambda_{\phi, w}$ if $m(\operatorname{supp}(z))<\infty$ (or respectively, $m(\operatorname{supp}(z))=\infty)$, then there is (cf. [2]) a measure preserving transformation $\sigma: \mathbb{R}^{+} \rightarrow \mathbb{R}^{+}$(respectively, $\sigma: \operatorname{supp}(z) \rightarrow \mathbb{R}_{+}$) such that

(i) $\int_{0}^{\infty} \phi(z) w \circ \sigma=\int_{0}^{\infty} \phi\left(z^{*}\right) w$;

(ii) if $|z(t)|<|z(s)|$, then $\sigma(t) \geq \sigma(s)$.

For a Lorentz-Orlicz space $\Lambda_{\phi, w}(\Omega), \phi$ is said to satisfy the $\Delta_{2}$ condition if one of the following holds:

(iii) $\Omega=[0, \infty)$ and there exists $l>0$ such that $\phi(2 u) \leq l \phi(u)$ for all $u>0$.

(iv) $\Omega=[0,1]$ and there are $l>0$ and $u_{0}>0$ such that $\phi(2 u) \leq l \phi(u)$ for all $u \geq u_{0}$.

In [7], Kamińska proved the following theorem.

Theorem A. For a Lorentz-Orlicz space $\Lambda_{\phi, w}$, the following are equivalent:

(1) $\Lambda_{\phi, w}$ is order continuous. So the Köthe dual of $\Lambda_{\phi, w}$ is the dual of $\Lambda_{\phi, w}$.

(2) $\Lambda_{\phi, w}$ does not contain any isometric copy of $\ell_{\infty}$.

(3) $\phi$ satisfies the $\Delta_{2}$ condition and $\int_{0}^{\infty} w=\infty$ if $\Omega=(0, \infty)$.

(4) For any $x \in \Lambda_{\phi, w}, \varrho_{\phi}(x)=1$ if and only if $\|x\|=1$.

Let $X$ be a Banach space. For any bounded subset $A$ of $X$, define

$$
\begin{aligned}
r(x, A) & =\sup \{\|x-y\|: y \in A\} \quad \text { for any } x \in A ; \\
R(A) & =\inf \{r(x, A): x \in A\} ; \\
\delta(A) & =\sup \{r(x, A): x \in A\}=\operatorname{diam} A .
\end{aligned}
$$

A bounded closed convex set $A$ is said to have normal structure if for any closed convex subset $B$ of $A$ either $R(B)=0$ or $R(B)<\delta(B)$. $X$ is said to have (weakly) normal structure if every bounded (weakly compact) closed convex subset of $X$ has normal structure. Kirk [9] showed that every nonexpansive mapping on a weakly compact convex set with normal structure has the fixed point property. 
Recall that a sequence $\left\{x_{n}\right\}$ in $X$ is said to be a limit-constant sequence if for any $x \in \operatorname{co}\left\{x_{n}\right\}$,

$$
\lim _{n \rightarrow \infty}\left\|x-x_{n}\right\|=\operatorname{diam}\left\{x_{n}\right\} .
$$

Note that here we require the limit to converge to the diameter of $\operatorname{co}\left\{x_{n}\right.$ : $n \in \mathbb{N}\}$ (cf. [10]). A sequence $\left\{x_{n}\right\}$ is said to be a unit limit-constant sequence if $\left\{x_{n}\right\}$ is a limit-constant sequence with $\operatorname{diam}\left\{x_{n}\right\}=1$. It is known that a Banach space $X$ has (weakly) normal structure if and only if $X$ contains no (weakly convergent) unit limit-constant sequence [10]. In [3], Chen showed that if $\phi$ is an $N$-function (for definition see [3]) which satisfies the $\Delta_{2}$ condition, then the Orlicz space $L_{\phi}$ has weakly normal structure. Recently, Carothers, Dilworth, Hsu, Lennard and Trautman [1, 5] studied the uniform Kadec-Klee property for the Lorentz space $L_{w, 1}$. They proved that $L_{w, 1}$ does not have normal structure and they also gave a sufficient condition for $L_{w, 1}$ to have weakly normal structure. In this article, we study (weakly) normal structure for Lorentz-Orlicz spaces and give a characterization of the Lorentz-Orlicz spaces with (weakly) normal structure. For more results about normal structure of Orlicz function (respectively, sequence) spaces and Lorentz function spaces, see [1, 3, 5, 6, 8, and 11].

It is known that $L_{1}$ does not have weakly normal structure and $\ell_{\infty}$ contains an isometric copy of $L_{1}$. Hence $\Lambda_{\phi, w}$ does not have weakly normal structure if $\Lambda_{\phi, w}$ is not order continuous. For a fixed Young function $\phi$ : $\mathbb{R} \rightarrow \mathbb{R}_{+} \cup\{0\}$ and a fixed weight function $w$, let $u_{0}$ and $v_{0}$ be defined by

$$
\begin{aligned}
& u_{0}=\sup \{u: \phi \text { is linear on }(0, u)\}, \\
& v_{0}=\sup \{v: w \text { is constant on }(0, v)\},
\end{aligned}
$$

where $\sup \emptyset=0$. The following are three examples of unit limit-constant sequences in Lorentz-Orlicz spaces. The first two are well-known.

Example 1 [1]. Suppose that $\phi$ is linear on $(0, \infty)$ and $a_{n}$ is the number such that

$$
\phi(n) \int_{0}^{a_{n}} w(t) d t=\frac{1}{2}
$$

Let $e_{n}=n 1_{\left(0, a_{n}\right)}$. It is easy to see that $\left\{e_{n}\right\}$ is a unit limit-constant sequence. So if $\Lambda_{\phi, w}(0,1)$ has normal structure, then $u_{0}<\infty$.

Suppose that $\Omega=(0, \infty)$ and $\phi$ is linear on $\left(0, u_{0}\right)$ for some $u_{0}>0$. Let $b_{n}$ be the number such that

$$
\phi\left(\frac{u_{0}}{n}\right) \int_{0}^{b_{n}} w(t) d t=\frac{1}{2} .
$$

A similar proof shows that $\left\{e_{n}=\left(u_{0} / n\right) 1_{\left(0, b_{n}\right)}\right\}$ is a unit limit-constant sequence. Hence if $\Lambda_{\phi, w}(0, \infty)$ has normal structure, then $u_{0}=0$. 
EXAmPle 2. Suppose that there exist two positive numbers $u$ and $v$ such that $\phi$ is linear on $(0, u), w$ is constant on $(0, v)$, and $\int_{0}^{v} \phi(u / 2) w \geq 1$. Without loss of generality, we may assume that $\int_{0}^{v} \phi(u / 2) w=1$. Let

$$
x_{n}(t)= \begin{cases}\frac{u}{2} \cdot \operatorname{sgn}\left(\sin \left(\frac{2^{n} \pi t}{v}\right)\right) & \text { if } t \leq v, \\ 0 & \text { otherwise. }\end{cases}
$$

Then for any $x \in \overline{\operatorname{co}}\left\{x_{i}: i \leq k\right\}$ and $n>k,\left\|x-x_{n}\right\|=1$. This implies that $\left\{x_{n}\right\}$ is a unit limit-constant sequence. It is known that $\Lambda_{\phi, w}(0, v)$ is not equal to $L_{\infty}(0,1)$ up to equivalent norm. By Proposition 2.c.10 in [13] (p. 160), $\left\{x_{n}\right\}$ is a weakly null sequence. Hence if $\Lambda_{\phi, w}$ has weakly normal structure, then $\int_{0}^{v_{0}} \phi\left(u_{0}\right) \cdot w<2$.

EXAmple 3. Suppose that $u_{0}>0$ and for some $v>0, w$ is constant on $(v, \infty)$. Then there are $0<u<u_{0}$ and $v^{\prime}>v$ such that

$$
\int_{0}^{2 v^{\prime}} \phi(u) w=1 .
$$

Let $e_{n}=u 1_{\left((n-1) v^{\prime}, n v^{\prime}\right)}$. If $a_{k} \geq 0$ and $\sum_{k=1}^{N} a_{k}=1$, then

$$
\begin{aligned}
\varrho_{\phi}\left(e_{N+1}-\sum_{k=1}^{N} a_{k} e_{k}\right) & =\int_{0}^{v^{\prime}} \phi(u) w(t) d t+\sum_{k=1}^{N} \int_{k v^{\prime}}^{(k+1) v^{\prime}} \phi\left(a_{k} u\right) w(t) d t \\
& =\int_{0}^{2 v^{\prime}} \phi(u) w=1 .
\end{aligned}
$$

So $\left\{e_{n}\right\}$ is a unit limit-constant sequence.

We claim that $\left\{e_{n}\right\}$ is equivalent to the natural basis of $\ell_{1}$. So it cannot be a weakly convergent sequence.

In fact, for any finite sequence $\left\{a_{k}\right\}_{k=1}^{N}$ with

$$
\sum_{k=1}^{N}\left|a_{k}\right| \geq \frac{1}{\int_{v^{\prime}}^{2 v^{\prime}} \phi(u) w}
$$

we have

$$
\varrho_{\phi}\left(\sum_{k=1}^{N} a_{k} e_{k}\right) \geq \sum_{k=1}^{N}\left|a_{k}\right| \int_{v^{\prime}}^{2 v^{\prime}} \phi(u) w \geq 1 .
$$

Hence

$$
\left\|\sum_{k=1}^{N} a_{k} e_{k}\right\| \geq \frac{\sum_{k=1}^{N}\left|a_{k}\right|}{\int_{v^{\prime}}^{2 v^{\prime}} \phi(u) w}
$$

This implies that $\left\{e_{n}\right\}$ is equivalent to the natural basis of $\ell_{1}$. 
From the above examples, it is natural to ask the following questions:

(1) Does $\Lambda_{\phi, w}(0, \infty)$ (respectively, $\Lambda_{\phi, w}(0,1)$ ) have normal structure if $u_{0}=0$ (respectively, $u_{0}<\infty$ and $\int_{0}^{v_{0}} \phi\left(u_{0}\right) \cdot w<2$ )?

(2) Does $\Lambda_{\phi, w}$ have weakly normal structure if $\int_{0}^{v_{0}} \phi\left(u_{0}\right) w<2$ ?

The following theorem shows that the answer to the above questions is affirmative.

Theorem 1. Suppose that $\Lambda_{\phi, w}$ is an order continuous Lorentz-Orlicz space.

(1) $\Lambda_{\phi, w}$ has normal structure if $u_{0}=0$ (respectively, $\int_{0}^{v_{0}} \phi\left(u_{0}\right) w<2$ and $\left.u_{0}<\infty\right)$.

(2) $\Lambda_{\phi, w}$ has weakly normal structure if $\int_{0}^{v_{0}} \phi\left(u_{0}\right) w<2$.

2. Basic properties of unit limit-constant sequences in $\Lambda_{\phi, w}$. First, we need the following three lemmas. The first one easily follows from the definition and the second one was proved in [12].

Lemma 2. Suppose that $v>\varepsilon>0$ and $u_{2}>u_{1}>0$. If $x$ is an element of $\Lambda_{\phi, w}$ such that

$$
m\left(\left\{t \in(0, v):|x(t)| \leq u_{1}\right\}\right)>\varepsilon, \quad m\left(\left\{t \in(v, \infty):|x(t)| \geq u_{2}\right\}\right)>\varepsilon,
$$

then

$$
\int \phi(|x|) w \leq \varrho_{\phi}(x)-\left(\phi\left(u_{2}\right)-\phi\left(u_{1}\right)\right)\left(\int_{v-\varepsilon}^{v} w-\int_{v}^{v+\varepsilon} w\right) .
$$

R e m ark 1. Suppose that either $w$ is not constant on $(v-\varepsilon, v)$ or $w$ is not constant on $(v, v+\varepsilon)$. Then $\int_{v-\varepsilon}^{v} w-\int_{v}^{v+\varepsilon} w>0$. Hence there is $\delta>0$ such that $\varrho_{\phi}(x) \geq \delta+\int \phi(x) w$ whenever $x$ satisfies the assumption of Lemma 2 .

Lemma 3. Let $\Lambda_{\phi, w}$ be an order continuous Lorentz-Orlicz space and $E$ be a set of positive measure and $\lambda$ be a positive number. Suppose that $x, y$ and $z$ are three elements of $\Lambda_{\phi, w}$ such that $\varrho_{\phi}(x-y) \leq 1, \varrho_{\phi}(x-z) \leq 1$ and

$$
\phi\left(x(t)-\frac{1}{2}(y(t)+z(t))\right) \leq \frac{\phi(x(t)-y(t))+\phi(x(t)-z(t))}{2}-\lambda
$$

for every $t \in E$. Then there is $\nu>0$ such that

$$
\varrho_{\phi}\left(x-\frac{y+z}{2}\right) \leq 1-\nu .
$$

Lemma 4. Let $\phi$ be a Young function. For any given $\delta>0$, there exists $\varepsilon>0$ such that

$$
\phi\left(d_{2}-\frac{d_{1}}{2}\right)<\frac{1}{2}\left(\phi\left(d_{2}-d_{1}\right)+\phi\left(d_{2}\right)\right)
$$

whenever $d_{1}>u_{0}+\delta$ and $0<d_{2}<d_{1}+\varepsilon$. 
Proof. If $u_{0}=0$, then there is $\varepsilon<\delta / 3$ such that $\phi$ is not linear on $(\varepsilon, 2 \varepsilon)$. If $u_{0}>0$, let $\varepsilon=\frac{1}{3} \min \left\{u_{0}, \delta\right\}$. Then $\phi$ is not linear on $\left(\varepsilon, u_{0}+\varepsilon\right)$. Hence if $d_{3}>2 \varepsilon+u_{0}$ and $0 \leq d_{4}<\varepsilon$, then

$$
\phi\left(\frac{d_{3}+d_{4}}{2}\right)<\frac{1}{2}\left(\phi\left(d_{3}\right)+\phi\left(d_{4}\right)\right) .
$$

Case $1: d_{1} \leq d_{2}$. In this case, $d_{2}-d_{1}<\varepsilon$, and $d_{2} \geq d_{1}>u_{0}+\delta>$ $u_{0}+2 \varepsilon$. So

$$
\phi\left(d_{2}-\frac{d_{1}}{2}\right)=\phi\left(\frac{d_{2}+d_{2}-d_{1}}{2}\right)<\frac{1}{2}\left(\phi\left(d_{2}-d_{1}\right)+\phi\left(d_{2}\right)\right) .
$$

Case 2: $d_{1}>d_{2}$. If $d_{2}<d_{1} / 2$, then

$$
\phi\left(d_{2}-\frac{d_{1}}{2}\right)=\phi\left(\frac{d_{1}}{2}-d_{2}\right) \leq \phi\left(\frac{d_{1}-d_{2}}{2}\right) \leq \frac{1}{2} \phi\left(d_{1}-d_{2}\right) .
$$

If $d_{2} \geq d_{1} / 2$, then

$$
\phi\left(d_{2}-\frac{d_{1}}{2}\right) \leq \phi\left(d_{2}-\frac{d_{2}}{2}\right)=\phi\left(\frac{d_{2}}{2}\right) \leq \frac{1}{2} \phi\left(d_{2}\right) .
$$

Hence

$$
\phi\left(d_{2}-\frac{d_{1}}{2}\right)<\frac{1}{2}\left(\phi\left(d_{2}-d_{1}\right)+\phi\left(d_{2}\right)\right) .
$$

It seems that the following proposition is known. But we cannot find a reference. So we present a proof.

Proposition 5. Let $\left\{x_{n}\right\}$ be a sequence in the unit ball of an order continuous Köthe space $E$ and $\left\{B_{n}\right\}$ be a sequence of disjoint measurable subsets. If $\left\{x_{n} 1_{B_{n}}\right\}$ is equivalent to the natural basis of $\ell_{1}$, then $\left\{x_{n}\right\}$ does not converge weakly.

Proof. Since $\left\{x_{n} 1_{B_{n}}\right\}$ is equivalent to the natural $\ell_{1}$ basis, there is $x^{*}$ in the dual of $\Lambda_{\phi, w}$ such that $\left\langle x^{*}, x_{n} 1_{B_{n}}\right\rangle=1$. We claim that

$$
\lim _{j \rightarrow \infty} \lim _{n \rightarrow \infty}\left\langle x^{*} 1_{B_{j}}, x_{n}\right\rangle=0 .
$$

By passing to further subsequences of $\left\{x_{n}\right\}$, we may assume that for any $j \in \mathbb{N}, \lim _{n \rightarrow \infty}\left\langle x^{*} 1_{B_{j}}, x_{n}\right\rangle$ exists. Suppose the claim is not true. Then there exist $c>0, L \geq\left\|x^{*}\right\| / c, l$ and $F \subseteq \mathbb{N}$ such that $\operatorname{card}(F) \geq L$ and for any $j \in F$,

$$
\left|\left\langle x^{*} 1_{B_{j}}, x_{l}\right\rangle\right|>c .
$$

This implies $\left\langle\left|x^{*}\right|,\left|x_{l}\right|\right\rangle>L c \geq\left\|x^{*}\right\|$, which contradicts $\left\|x_{l}\right\| \leq 1$.

We claim that there is a subsequence $\left\{x_{n_{k}}\right\}$ of $\left\{x_{n}\right\}$ such that

$$
\left|\left\langle x^{*} 1_{B_{n_{i}}}, x_{n_{l}}\right\rangle\right|<\frac{1}{4} \quad \text { for any } l \geq i+1 ;
$$




$$
\left\|x_{n_{i}} 1_{\cup_{j=n_{i}}^{\infty} B_{n_{j}}}\right\| \leq \frac{1}{4^{i+1}\left\|x^{*}\right\|} .
$$

By (1), there is $n_{1}$ such that

$$
\lim _{k \rightarrow \infty}\left|\left\langle x^{*} 1_{B_{n_{1}}}, x_{k}\right\rangle\right|<\frac{1}{4} .
$$

We can find an $n_{2}>n_{1}$ such that

$$
\begin{array}{rlrl}
\left|\left\langle x^{*} 1_{B_{n_{1}}}, x_{l}\right\rangle\right| & <\frac{1}{4} \quad & \text { for any } l \geq n_{2} ; \\
\left\|x_{n_{1}} 1_{\cup_{j=n_{2}}^{\infty} B_{n_{j}}}\right\| & \leq \frac{1}{4^{2}\left\|x^{*}\right\|} & & \text { (since } E \text { is order continuous); } \\
\lim _{k \rightarrow \infty}\left|\left\langle x^{*} 1_{B_{n_{2}}}, x_{k}\right\rangle\right| & <\frac{1}{4^{2}} \quad & \text { by }(1) .
\end{array}
$$

Assume that $n_{1}, \ldots, n_{i}$ are selected. Then there is $n_{i+1}>n_{i}$ such that

$$
\begin{aligned}
\left|\left\langle x^{*} 1_{B_{n_{i}}}, x_{l}\right\rangle\right| & <\frac{1}{4^{i}} & & \text { for any } l \geq n_{i+1} ; \\
\left\|x_{n_{i}} 1_{\cup_{j=n_{i+1}}^{\infty} B_{n_{j}}}\right\| & \leq \frac{1}{4^{i+1}\left\|x^{*}\right\|} & & \text { (since } E \text { is order continuous); } \\
\lim _{k \rightarrow \infty}\left|\left\langle x^{*} 1_{B_{n_{i+1}}}, x_{k}\right\rangle\right| & <\frac{1}{4^{i+1}} & & \text { by }(1) .
\end{aligned}
$$

We have constructed a subsequence $\left\{x_{n_{k}}\right\}$ which satisfies our claim. Let $\left\{a_{j}: 1 \leq j \leq N\right\}$ be any finite real sequence, and let

$$
E_{1}=\bigcup\left\{B_{j}: a_{j}>0 \text { and } j \leq N\right\}, \quad E_{2}=\bigcup\left\{B_{j}: a_{j} \leq 0 \text { and } j \leq N\right\} .
$$

Then

$$
\begin{aligned}
\left\|x^{*}\right\| \cdot\left\|\sum_{j=1}^{N} a_{j} x_{n_{j}}\right\| \geq & \left\langle x^{*} 1_{E_{1}}-x^{*} 1_{E_{2}}, \sum_{j=1}^{N} a_{j} x_{n_{j}}\right\rangle \\
\geq & \sum_{j=1}^{N}\left(\left|a_{j}\right|\left\langle x^{*}, 1_{B_{j}} x_{n_{j}}\right\rangle\right. \\
& \left.-\sum_{i=1}^{j-1}\left|a_{j}\right| \cdot\left|\left\langle x^{*}, 1_{B_{i}} x_{n_{j}}\right\rangle\right|-\left|a_{j}\right| \cdot\left\|x_{n_{j}} 1_{\cup_{l=j+1}^{\infty} B_{n_{l}}}\right\|\right) \\
\geq & \sum_{j=1}^{N}\left|a_{j}\right|\left(1-\sum_{i=1}^{j+1} \frac{1}{4^{i}}\right) \geq \frac{2}{3} \sum_{j=1}^{N}\left|a_{j}\right| .
\end{aligned}
$$

This implies that $\left\{x_{n_{k}}\right\}$ is equivalent to the natural basis of $\ell_{1}$. So $\left\{x_{n}\right\}$ cannot converge weakly.

Suppose that $\Lambda_{\phi, w}$ is an order continuous Lorentz-Orlicz function space without (weakly) normal structure. There exists a (weakly convergent) unit 
limit-constant sequence $\left\{x_{n}\right\}$ in $\Lambda_{\phi, w}$. (From now on, $\left\{x_{n}\right\}$ is a fixed (weakly convergent) unit limit-constant sequence.) Let

$$
\bar{x}_{n}=\frac{1}{n} \sum_{k=1}^{n} x_{k}, \quad z_{n}^{\prime}=\sup \left\{x_{1}, \ldots, x_{n}\right\}, \quad z_{n}^{\prime \prime}=\inf \left\{x_{1}, \ldots, x_{n}\right\} .
$$

Then $\left\{z_{n}^{\prime}\right\}$ is an increasing sequence. It converges in measure to an extended measurable function

$$
z^{\prime}=\sup \left\{x_{n}: n \in \mathbb{N}\right\} \equiv \lim _{n \rightarrow \infty} z_{n}^{\prime} .
$$

Similarly, $\left\{z_{n}^{\prime \prime}\right\}$ is a decreasing sequence, and it converges in measure to another extended measurable function

$$
z^{\prime \prime}=\inf \left\{x_{n}: n \in \mathbb{N}\right\} \equiv \lim _{n \rightarrow \infty} z_{n}^{\prime \prime} .
$$

Lemma $6 . \quad m\left(\left\{t:\left|z^{\prime}(t)-z^{\prime \prime}(t)\right|>u_{0}\right\}\right)=0$.

Pro of. If $u_{0}=\infty$, then there is nothing to be proved. So we may assume that $u_{0}<\infty$. Suppose that the lemma is not true. Since

$$
\left\{t:\left|z^{\prime}(t)-z^{\prime \prime}(t)\right|>u_{0}\right\}=\bigcup_{m, n \in \mathbb{N}}\left\{t:\left|x_{n}(t)-x_{m}(t)\right|>u_{0}\right\},
$$

there are $n$ and $m$ such that

$$
m\left(\left\{t:\left|x_{n}(t)-x_{m}(t)\right|>u_{0}\right\}\right)>0 .
$$

By passing to a subsequence, we may assume that $x_{n}=x_{1}$ and $x_{m}=x_{2}$. Let $A=\left\{t: x_{2}(t)-x_{1}(t) \geq 0\right\}$. Replacing $x_{k}$ by $\left(x_{k}-x_{1}\right) 1_{A}-\left(x_{k}-x_{1}\right) 1_{\Omega \backslash A}$, we may assume that $x_{2} \geq 0$. By measure theory, there is $\delta>0$ such that

$$
m\left(\left\{t:\left|x_{1}(t)-x_{2}(t)\right|>u_{0}+\delta\right\}\right)>c>0 .
$$

By Lemma 4, there is $\varepsilon>0$ such that

$$
\phi\left(d_{2}-\frac{d_{1}}{2}\right)<\frac{1}{2}\left(\phi\left(d_{2}-d_{1}\right)+\phi\left(d_{2}\right)\right)
$$

provided $d_{1}>u_{0}+\delta$ and $0<d_{2}<d_{1}+\varepsilon$.

Claim. There are a subsequence $\left\{y_{k}\right\}_{k=1}^{\infty}$ of $\left\{x_{n}\right\}$ and a decreasing sequence $\left\{C_{k}\right\}_{k=2}^{\infty}$ of measurable sets such that

(a) $y_{1}=x_{1}, y_{2}=x_{2}$;

(b) $m\left(C_{n}\right) \geq\left(1 / 2+1 / 2^{n}\right) c$;

(c) for any $t \in C_{n}$, there is $k<n$ such that

$$
\begin{aligned}
\left|y_{n}(t)-y_{k}(t)\right| & \geq \varepsilon+\sup \left\{\left|y_{n-1}(t)-y_{j}(t)\right|: j<n\right\} \\
& =\varepsilon+\sup \left\{\left|y_{i}(t)-y_{j}(t)\right|: i, j<n\right\} .
\end{aligned}
$$



then

Suppose the claim were proved. Note that if $n>2, t \in C_{n}$ and $y_{n}(t)>0$,

$$
y_{n}(t)-\inf \left\{y_{i}(t): 1 \leq i \leq n-1\right\} \geq \varepsilon+\sup \left\{\left|y_{n-1}(t)-y_{i}(t)\right|: 1 \leq i<n\right\} .
$$

Similarly, if $y_{n}(t)<0$, then

$$
\sup \left\{y_{i}(t): 1 \leq i \leq n-1\right\}-y_{n}(t) \geq \varepsilon+\sup \left\{\left|y_{n-1}(t)-y_{i}(t)\right|: 1 \leq i<n\right\} .
$$

So for any $t \in C_{n}$ and $k<m \leq n-1$, we have

$$
\left(y_{n}(t)-y_{m}(t)\right)\left(y_{n}(t)-y_{k}(t)\right) \geq 0 \text {, }
$$

and

$$
\begin{aligned}
\left|y_{n}(t)-y_{m}(t)\right| \geq & \sup \left\{\left|y_{n}(t)-y_{j}(t)\right|: j \leq n-1\right\} \\
& -\sup \left\{\left|y_{m}(t)-y_{j}(t)\right|: j \leq n-1\right\} \geq \varepsilon .
\end{aligned}
$$

This implies

$$
\operatorname{card}\left(\left\{j \leq n-1:\left|y_{n}(t)-y_{j}(t)\right|<l \varepsilon\right\}\right) \leq l-1,
$$

and

$$
\left|y_{n}(t)-\bar{y}_{n-1}(t)\right|=\frac{1}{n-1} \sum_{i=1}^{n-1}\left|y_{n}(t)-y_{i}(t)\right| \geq \frac{1}{n-1} \sum_{i=1}^{n-1} i \varepsilon=\frac{n \varepsilon}{2} .
$$

Therefore,

$$
\int_{\Omega} \phi\left(\left(y_{n}-\bar{y}_{n-1}\right)^{*}\right) w \geq \int_{0}^{m\left(C_{n}\right)} \phi\left(\frac{n \varepsilon}{2}\right) w \geq \phi\left(\frac{n \varepsilon}{2}\right) \int_{0}^{c / 2} w,
$$

which is impossible if $n$ is large enough. Hence the lemma must be true.

Proof of Claim. Let $C_{2}=\left\{t:\left|y_{1}(t)-y_{2}(t)\right|>u_{0}+\delta\right\}$. (So $m\left(C_{2}\right)<$ $\infty$.) Suppose that $y_{1}, \ldots, y_{k}=x_{n_{k}}$ and $C_{2}, \ldots, C_{k}$ have been constructed. For $j<k$, let

$$
\begin{aligned}
D_{j}=\left\{t \in C_{k}\right. & :\left|y_{k}(t)-y_{j}(t)\right| \\
& \left.=\sup \left\{\left|y_{k}(t)-y_{i}(t)\right|: i<k\right\}>\sup \left\{\left|y_{k}(t)-y_{i}(t)\right|: i<j\right\}\right\} .
\end{aligned}
$$

Then $C_{k}=\bigcup_{j=1}^{k-1} D_{j}$.

SubClaim. There is $M_{j}>n_{k}$ such that for any $n \geq M_{j}$,

$$
\begin{aligned}
m\left(\left\{t \in D_{j}:\right.\right. & \sup \left\{\left|x_{n}(t)-y_{i}(t)\right|: i \leq k\right\} \\
& \left.\left.\geq \sup \left\{\left|y_{k}(t)-y_{i}(t)\right|: i<k\right\}+\varepsilon\right\}\right) \geq\left(1-1 / 2^{k+1}\right) m\left(D_{j}\right) .
\end{aligned}
$$

Suppose that the subclaim were proved. Let $n_{k+1}=\sup \left\{M_{j}: j<k\right\}$, $y_{k+1}=x_{n_{k+1}}$, and

$$
\begin{aligned}
C_{k+1}=\left\{t \in C_{k}:\right. & \sup \left\{\left|y_{k+1}(t)-y_{j}(t)\right|: j \leq k\right\} \\
& \left.\geq \sup \left\{\left|y_{k}(t)-y_{j}(t)\right|: j<k\right\}+\varepsilon\right\} .
\end{aligned}
$$

Then $C_{k+1}$ and $y_{k+1}$ satisfy (b) and (c), hence the claim is proved. 
Proof of Subclaim. If $m\left(D_{j}\right)=0$, then let $M_{j}=n_{k}+1$. So we may assume that $m\left(D_{j}\right)>0$. By measure theory, there exists $L>\delta+u_{0}$ such that

$$
m\left(\left\{t \in D_{j}:\left|y_{k}(t)-y_{j}(t)\right| \leq L\right\}\right)>\left(1-1 / 2^{k+2}\right) m\left(D_{j}\right)
$$

for any $m\left(D_{j}\right)>0, j \leq k$. Note that if $t \in D_{j}$, then $u_{0}+\delta<\left|y_{k}(t)-y_{j}(t)\right|$. Suppose the subclaim is not true. Then for any $N>n_{k}$, there is $m>N$ such that

$$
\begin{aligned}
E_{m, j}=\left\{t \in D_{j}:\right. & \max \left\{\left|x_{m}(t)-y_{k}(t)\right|,\left|x_{m}(t)-y_{j}(t)\right|\right\} \\
& \left.<\left|y_{k}(t)-y_{j}(t)\right|+\varepsilon \text { and } u_{0}+\delta<\left|y_{k}(t)-y_{j}(t)\right| \leq L\right\}
\end{aligned}
$$

has measure greater than $2^{-(k+2)} m\left(D_{j}\right)$. For any $t \in E_{m, j}$, either $y_{k}(t)>$ $y_{j}(t)$ or $y_{k}(t)<y_{j}(t)$. Without loss of generality, we assume that $y_{k}(t)>$ $y_{j}(t)$ and $y_{k}(t)+\varepsilon \geq x_{m}(t) \geq y_{j}(t)-\varepsilon$. Let $d_{1}=y_{k}(t)-y_{j}(t)$ and

$$
d_{2}= \begin{cases}y_{k}(t)-x_{m}(t) & \text { if } x_{m}(t) \leq y_{k}(t), \\ x_{m}(t)-y_{j}(t) & \text { otherwise. }\end{cases}
$$
that

Since $[0, L]$ is compact and $\phi$ is continuous, by (2), there is $\lambda>0$ such

$$
\phi\left(d_{2}-\frac{d_{1}}{2}\right) \leq \frac{1}{2}\left(\phi\left(d_{2}-d_{1}\right)+\phi\left(d_{2}\right)\right)-\lambda
$$

whenever $L \geq d_{1}>u_{0}+\delta$ and $d_{2} \leq d_{1}+\varepsilon$. So

$$
\begin{aligned}
\phi\left(\frac{1}{2}\left(y_{k}(t)+y_{j}(t)\right)-\right. & \left.x_{m}(t)\right) \\
& =\phi\left(y_{k}(t)-x_{m}(t)-\frac{y_{k}(t)-y_{j}(t)}{2}\right) \\
& \leq \frac{1}{2}\left(\phi\left(y_{k}(t)-x_{m}(t)\right)+\phi\left(y_{j}(t)-x_{m}(t)\right)\right)-\lambda .
\end{aligned}
$$

Note that $\varrho_{\phi}\left(x_{m}-\frac{1}{2}\left(y_{j}+y_{k}\right)\right) \leq 1$ and $\int_{0}^{\infty} w=\infty$. By Lemma 3 , there is $\nu>0$ (which depends on $\lambda, w$ and $m\left(D_{j}\right)$, but is independent of $x_{m}$ ) such that

$$
\int_{0}^{\infty} \phi\left(\left(x_{m}-\frac{1}{2}\left(y_{j}+y_{k}\right)\right)^{*}\right) w \leq 1-\nu .
$$

Since $\phi$ satisfies the $\Delta_{2}$ condition, by Theorem A,

$$
\liminf _{m \rightarrow \infty}\left\|x_{m}-\frac{1}{2}\left(y_{k}+y_{j}\right)\right\|<1,
$$

which contradicts the fact that $\left\{x_{n}\right\}$ is a unit limit-constant sequence. So the subclaim must be true and the proof of Lemma 6 is complete.

Remark 2. Since $\left\{x_{n}\right\}$ is not a constant sequence, we have $u_{0}>0$. 
Lemma 7. For any $l \in \mathbb{N}$ and $\varepsilon>0$,

$$
\lim _{n \rightarrow \infty} m\left(\left\{t: z_{l}^{\prime \prime}(t)+\varepsilon<x_{n}(t)<z_{l}^{\prime}(t)-\varepsilon\right\}\right)=0 .
$$

Pro of. Suppose the lemma is not true. By passing to a further subsequence of $\left\{x_{n}\right\}$, we may assume that there are $\varepsilon>0$ and $\delta>0$ such that for all $m>l$, the set

$$
F_{m}=\left\{t: z_{l}^{\prime \prime}(t)+\varepsilon<x_{m}(t)<z_{l}^{\prime}(t)-\varepsilon\right\}
$$

has measure at least $\delta$. Let $\sigma$ be a measure preserving transformation such that

(i) $\int_{0}^{\infty} \phi\left(x_{m}-\bar{x}_{l}\right) w \circ \sigma=\int_{0}^{\infty} \phi\left(\left(x_{m}-\bar{x}_{l}\right)^{*}\right) w$;

(ii) if $\left|\left(x_{m}-\bar{x}_{l}\right)(t)\right|<\left|\left(x_{m}-\bar{x}_{l}\right)(s)\right|$, then $\sigma(t) \geq \sigma(s)$.

Since for any $t \in F_{m}$,

$$
\frac{1}{l} \sum_{k=1}^{l}\left|x_{m}(t)-x_{k}(t)\right| \geq \frac{\varepsilon}{l}+\left|x_{m}(t)-\bar{x}_{l}(t)\right|,
$$

by Lemma 3, there is $\nu>0$ (dependent only on $l, \delta$ and $\varepsilon$ ) such that

$$
\begin{aligned}
\int_{0}^{\infty} \phi\left(\left(x_{m}-\bar{x}_{l}\right)^{*}\right) w & =\int_{0}^{\infty} \phi\left(x_{m}-\frac{1}{l} \sum_{k=1}^{l} x_{k}\right) w \circ \sigma \\
& \leq \frac{1}{l} \sum_{k=1}^{l} \varrho_{\phi}\left(\left|x_{m}-x_{k}\right|\right)-\nu \leq 1-\nu .
\end{aligned}
$$

This contradicts $\lim _{m \rightarrow \infty}\left\|x_{m}-\bar{x}_{l}\right\|=1$.

Lemma 8. Suppose that there are two positive numbers $v_{1}, u_{1}$ such that

(1) either $w(t)<w\left(v_{1}\right)$ for all $t>v_{1}$ or $w(t)>w\left(v_{1}\right)$ for all $t<v_{1}$;

(2) for any $i \neq j, m\left(\left\{t:\left|x_{i}(t)-x_{j}(t)\right| \geq u_{1}\right\}\right) \leq v_{1}$.

Then for any $u_{2}>u_{1}, m\left(\left\{t: z^{\prime}(t)-z^{\prime \prime}(t) \geq u_{2}\right\}\right) \leq v_{1}$.

Proof. Since the proofs are similar, we can assume that $w(t)<w\left(v_{1}\right)$ for all $t>v_{1}$. Suppose that the lemma is not true. There is $\nu>0$ such that $u_{2}-u_{1}>2 \nu$ and

$$
m\left(\left\{t: z^{\prime}(t)-z^{\prime \prime}(t)>u_{1}+2 \nu\right\}\right)>v_{1}+2 \nu .
$$

Let

$$
F_{l}=\left\{t: z_{l}^{\prime}(t)-z_{l}^{\prime \prime}(t)>u_{1}+3 \nu / 2\right\} .
$$

Clearly, $m\left(F_{k}\right)<\infty$ for all $k \in \mathbb{N}$. Since $\left\{F_{k}\right\}$ is an increasing sequence and $\bigcup_{k=1}^{\infty} F_{k} \supseteq\left\{t: z^{\prime}(t)-z^{\prime \prime}(t)>u_{1}+2 t\right\}$, there is $l$ such that $m\left(F_{l}\right) \geq v_{1}+3 \nu / 2$. Let

$$
G_{n}=\left\{t \in F_{l}: x_{n}(t) \geq z_{l}^{\prime}(t)-\nu / 4 \text { or } x_{n}(t) \leq z_{l}^{\prime \prime}(t)+\nu / 4\right\}
$$


By Lemma $6, \lim _{n \rightarrow \infty} m\left(F_{l} \backslash G_{n}\right)=0$. So there is $N_{1}>l$ such that if $n>N_{1}$, then $m\left(G_{n}\right) \geq v_{1}+\nu$. This implies that for any measure preserving transformation $\sigma$ of $\Omega$,

$$
m\left(\left\{t \in G_{n}: t \in \sigma^{-1}\left(v_{1}, \infty\right)\right\}\right) \geq \nu .
$$

Fix $n>N_{1}$. By the definition of $G_{n}$, for any $t \in G_{n}$, either $x_{n}(t) \geq z_{l}^{\prime}(t)-$ $\nu / 4$ or $x_{n}(t) \leq z_{l}^{\prime \prime}(t)+\nu / 4$. Without loss of generality, $x_{n}(t) \geq z_{l}^{\prime}(t)-\nu / 4$. Let $j \leq l$ such that $x_{j}(t)=z_{l}^{\prime \prime}(t)$. Then

$$
\left|x_{n}(t)-x_{j}(t)\right| \geq z_{l}^{\prime}(t)-z_{l}^{\prime \prime}(t)-\nu / 4>u_{1}+5 \nu / 4 .
$$

Note that $\left\{x_{n}\right\}$ is a unit limit-constant sequence. For any $\lambda>0$, there are $n>N_{1}$ and a measure preserving transformation $\sigma$ such that

(i) $\int \phi\left(x_{n}-\bar{x}_{l}\right) w \circ \sigma=\int \phi\left(\left(x_{n}-\bar{x}_{l}\right)^{*}\right) w \geq 1-\lambda$;

(ii) if $\left|\left(x_{n}-\bar{x}_{l}\right)(t)\right| \geq\left|\left(x_{n}-\bar{x}_{l}\right)(s)\right|$, then $\sigma(t) \geq \sigma(s)$.

For any $k \leq l$, let

$$
H_{k}=\sigma^{-1}\left(v_{1}, \infty\right) \cap\left\{t:\left|x_{n}(t)-x_{k}(t)\right|>u_{1}+5 \nu / 4\right\} .
$$

Clearly, $\bigcup_{k=1}^{l} H_{k} \supseteq\left\{t \in G_{n}: t \in \sigma^{-1}\left(v_{1}, \infty\right)\right\}$. Hence there is $k \leq l$ such that $m\left(H_{k}\right) \geq \nu / l$. By $(2)$, the set $\left\{t \in \sigma^{-1}\left(0, v_{1}\right):\left|x_{n}(t)-x_{k}(t)\right|<u_{1}\right\}$ has measure at least $m\left(H_{k}\right)$. By Lemma 2 and Remark 1 , there is $\delta>0$ such that $\delta$ is only dependent on $u_{1}, \nu, v_{1}, l$, and

$$
\int \phi\left(\left|x_{n}(t)-x_{k}(t)\right|\right) w \circ \sigma(t) d t \leq \varrho_{\phi}\left(x_{n}-x_{k}\right)-\delta .
$$

This implies, for any $\lambda>0$,

$$
\begin{aligned}
1-\lambda \leq \int \phi\left(x_{n}-\bar{x}_{l}\right) w \circ \sigma & \leq \frac{1}{l} \sum_{j=1}^{l} \int \phi\left(x_{n}-x_{j}\right) w \circ \sigma \\
& \leq \frac{1}{l} \sum_{j=1}^{l} \varrho_{\phi}\left(x_{n}-x_{j}\right)-\frac{\delta}{l} \leq 1-\frac{\delta}{l} .
\end{aligned}
$$

It is impossible if $\lambda<\delta / l$.

We have the following two corollaries.

Corollary 9. If $v_{0}=0$, then $z^{\prime}$ and $z^{\prime \prime}$ are finite almost everywhere.

Proof. Since $v_{0}=0$ and $w$ is left continuous, for any $\delta>\delta_{1}>0$, there are $0<\delta_{2}<\delta_{1}$ and $u_{1}>0$ such that $\varrho_{\phi}\left(u_{1} 1_{\left(0, \delta_{2}\right)}\right)>1$ and $w(t)<w\left(\delta_{2}\right)$ if $t>\delta_{2}$. Since $\left\{x_{n}\right\}$ is a unit limit-constant sequence, for any $m, n$ we have $\varrho_{\phi}\left(x_{m}-x_{n}\right) \leq 1$. So

$$
m\left(\left\{t:\left|x_{n}(t)-x_{m}(t)\right|>u_{1}\right\}\right) \leq \delta_{2} \quad \text { for all } n, m \in \mathbb{N} .
$$

By Lemma 8, we have

$$
m\left(\left\{t: z^{\prime}(t)-z^{\prime \prime}(t) \geq u_{2}\right\}\right) \leq \delta_{2}
$$


for any $u_{2}>u_{1}$. Since $\delta_{2}$ is arbitrary, $z^{\prime}$ and $z^{\prime \prime}$ are finite almost everywhere.

Corollary 10. Suppose that $w$ is not constant on $(v, \infty)$ for any $v>0$. Then for any $\varepsilon>0$,

$$
m\left(\left\{t: z^{\prime}(t)-z^{\prime \prime}(t)>2 \varepsilon\right\}\right)<\infty .
$$

Proof. Since $\int_{0}^{\infty} w=\infty$ and $w$ is not constant on $(v, \infty)$ for any $v>0$, it follows that for any $\varepsilon>0$, there is $L>0$ such that $w(t)>w(L)$ for all $t>L$ and

$$
m\left\{t:\left|x_{n}(t)-x_{m}(t)\right| \geq \varepsilon\right\}<L
$$

for all $n, m$. By Lemma 8 , we have

$$
m\left(\left\{t: z^{\prime}(t)-z^{\prime \prime}(t)>2 \varepsilon\right\}\right)<L<\infty .
$$

Proposition 11. Suppose that there is $1>\delta>0$ such that one of the following conditions holds:

(1) For any $M>0$, there is $n$ such that $\varrho_{\phi}\left(x_{n} 1_{\left\{t:\left|x_{n}(t)\right|>M\right\}}\right)>\delta$.

(2) For any $\varepsilon>0$ there is $n$ such that $\varrho_{\phi}\left(x_{n} 1_{\left\{t:\left|x_{n}(t)\right|<\varepsilon\right\}}\right)>\delta$.

Then $\left\{x_{n}\right\}$ does not converge weakly.

P r o of. Since the proofs are similar, we only prove the proposition when (1) holds.

Suppose the proposition is not true. Then there is a weakly convergent unit limit-constant sequence $\left\{x_{n}\right\}$ satisfying (1). Lemma 6 yields $u_{0}=\infty$. By assumption, there exist sequences $\left\{D_{k}\right\},\left\{d_{k}\right\}$ and $\left\{n_{k}\right\}$ such that for all $k \in \mathbb{N}$ we have $8^{k} d_{k}<8^{k} D_{k}<\delta d_{k+1}<\delta D_{k+1}$ and $\varrho_{\phi}\left(x_{n_{k}} 1_{\left\{t: d_{k} \leq\left|x_{n_{k}}(t)\right| \leq D_{k}\right\}}\right)$ $>\delta$. Let

$$
A_{k}=\left\{t: d_{k} \leq\left|x_{n_{k}}(t)\right| \leq D_{k}\right\}, \quad B_{k}=A_{k} \backslash \bigcup_{j=k+1}^{\infty} A_{j} .
$$

Since $\varrho_{\phi}\left(x_{n_{k}}\right) \leq 1$ and $\left|x_{n_{k}}(t)\right| \geq d_{k}$ for every $t \in A_{k}, \int_{0}^{m\left(A_{k}\right)} w(t) d t \leq$ $1 / \phi\left(d_{k}\right)$. So

$$
\begin{aligned}
\varrho_{\phi}\left(x_{n_{k}} 1_{B_{k}}\right) & \geq \varrho_{\phi}\left(x_{n_{k}} 1_{A_{k}}\right)-\sum_{j=k+1}^{\infty} \varrho_{\phi}\left(x_{n_{k}} 1_{A_{j}}\right) \\
& \geq \delta-\sum_{j=k+1}^{\infty} \phi\left(D_{k}\right) \frac{1}{\phi\left(d_{j}\right)} \geq \delta-\frac{\delta}{3}=\frac{2 \delta}{3} .
\end{aligned}
$$

We claim that $\left\{x_{n_{k}} 1_{B_{k}}\right\}$ is equivalent to the natural basis of $\ell_{1}$. Without loss of generality, we assume that 


$$
B_{k}=\left(\sum_{j=k+1}^{\infty} m\left(B_{j}\right), \sum_{j=k}^{\infty} m\left(B_{j}\right)\right),
$$

and $\left|x_{n_{k}}\right| 1_{B_{k}}$ is decreasing on $B_{k}$. Then

$$
\begin{aligned}
\int \phi\left(x_{n_{k}} 1_{B_{k}}\right) w & \\
& =\int_{B_{k}} \phi\left(x_{n_{k}}\right) w \\
& =\int_{0}^{m\left(B_{k}\right)} \phi\left(x_{n_{k}}\right)\left(t+\sum_{j=k+1}^{\infty} m\left(B_{j}\right)\right) w\left(t+\sum_{j=k+1}^{\infty} m\left(B_{j}\right)\right) \\
& \geq \int_{0}^{m\left(B_{k}\right)} \phi\left(x_{n_{k}}\right)\left(t+\sum_{j=k+1}^{\infty} m\left(B_{j}\right)\right) w(t)-\phi\left(D_{k}\right) \sum_{j=k+1}^{\infty} 1 / \phi\left(d_{j}\right) \\
& \geq \varrho_{\phi}\left(x_{n_{k}} 1_{B_{k}}\right)-\frac{\delta}{3} \geq \frac{2 \delta}{3}-\frac{\delta}{3}=\frac{\delta}{3} .
\end{aligned}
$$

Hence, for any sequence $\left\{a_{n}\right\} \in \ell_{1}$ with $\sum_{n=1}^{\infty}\left|a_{n}\right| \geq 1 /(3 \delta)$,

$$
\begin{aligned}
\varrho_{\phi}\left(\sum_{j=1}^{\infty} a_{j} x_{n_{j}} 1_{B_{j}}\right) & \geq \int \phi\left(\sum_{j=1}^{\infty} a_{j} x_{n_{j}} 1_{B_{j}}\right) w=\sum_{j=1}^{\infty} \int_{B_{j}} \phi\left(a_{j} x_{n_{j}}\right) w \\
& =\sum_{j=1}^{\infty} \int_{B_{j}} a_{j} \phi\left(x_{n_{j}}\right) w \geq \sum_{j=1}^{\infty}\left|a_{j}\right| \frac{\delta}{3} \geq 1 .
\end{aligned}
$$

This implies that $\left\{x_{n_{k}} 1_{B_{k}}\right\}$ is equivalent to the natural basis of $\ell_{1}$. By Proposition 5, $\left\{x_{n}\right\}$ does not converge weakly.

Proposition 12. Suppose that for any $\nu>0$, there are a sequence $\left\{n_{i}\right\}$ and a measurable set $A$ such that $0<m(A) \leq v_{0}$ and

$$
\varrho_{\phi}\left(\left(x_{n_{k}}-x_{n_{j}}\right) 1_{A}\right) \geq 1-\nu \quad \text { whenever } i>j .
$$

Then $\int_{0}^{v_{0}} \phi\left(u_{0}\right) w \geq 2$.

Proof. It is clear that $v_{0}>0$. If $u_{0}=\infty$, then there is nothing to be proved. So we may assume that $u_{0}<\infty$. Replacing $x_{n}$ by $x_{n}-x_{1}$ if necessary, we may also assume that $x_{1} \equiv 0$. By Lemma 6 , both $z^{\prime}$ and $z^{\prime \prime}$ are bounded. Since $\phi$ is linear on $\left(0, v_{0}\right)$, without loss of generality, we further assume that $\phi(t)=t$ for all $0<t \leq u_{0}$ and $w(t)=1$ for all $t \leq v_{0}$. To prove the proposition, it is enough to show that $v_{0} \geq 2 / u_{0}$.

Let $K$ be a fixed natural number. For any $a<b$ and any $0 \leq l \leq 2 K$, let $\left\{a_{k}: 1 \leq k \leq 2 K\right\}$ be a finite sequence such that

$$
a_{k}= \begin{cases}a & \text { if } k \leq l \\ b & \text { otherwise }\end{cases}
$$


Then

$$
\sum_{i<j \leq 2 K}\left|a_{j}-a_{i}\right|=(2 K-l+1)(l-1)(b-a) \leq K^{2}(b-a) .
$$

Let $0<\delta<v_{0}$ be any positive number such that

$$
\int_{0}^{\delta} u_{0} d t \leq \frac{1}{K^{4}} .
$$

By assumption, there are a measurable set $A$ and a natural number $N$ such that $0<m(A) \leq v_{0}$ and

$$
\varrho_{\phi}\left(\left(x_{n_{k}}-x_{n_{j}}\right) 1_{A}\right) \geq 1-1 / K^{4} \quad \text { whenever } k>j \geq N \text {. }
$$

By the definition of $z^{\prime}$ and $z^{\prime \prime}$, there exists $l$ such that

$$
\begin{array}{r}
m\left\{t \in A:\left|z^{\prime}(t)-z_{l}^{\prime}(t)\right|>\frac{1}{2 K^{4} v_{0}}\right\}<\frac{\delta}{3}, \\
m\left\{t \in A:\left|z^{\prime \prime}(t)-z_{l}^{\prime \prime}(t)\right|>\frac{1}{2 K^{4} v_{0}}\right\}<\frac{\delta}{3} .
\end{array}
$$

By Lemma 7 , there exists a finite subsequence $\left\{k_{1}, \ldots, k_{2 K}\right\}$ of $\left\{n_{k}\right\}$ such that for any $j \leq 2 K$,

$$
m\left(\left\{t \in A: z_{l}^{\prime \prime}(t)+\frac{1}{2 K^{4} v_{0}}<x_{k_{j}}<z_{l}^{\prime}-\frac{1}{2 K^{4} v_{0}}\right\}\right)<\frac{\delta}{3} .
$$

Let

$$
B_{i}=\left\{t \in A:\left|z^{\prime \prime}(t)-x_{k_{i}}(t)\right| \geq \frac{1}{K^{4} v_{0}} \text { and }\left|x_{k_{i}}(t)-z^{\prime}(t)\right| \geq \frac{1}{K^{4} v_{0}}\right\} .
$$

Then for all $i \leq 2 K, B_{i}$ has measure at most $\delta$. For each $i \leq 2 K$, let $y_{i}$ be a measurable function such that $y_{i}(t) \in\left\{z^{\prime}(t), z^{\prime \prime}(t)\right\}$ and for any $t \in A \backslash B_{i}$, $\left|y_{i}(t)-x_{k_{i}}(t)\right|<\frac{1}{K^{4} v_{0}}$. Then

$$
\begin{aligned}
K(2 K-1)\left(1-\frac{1}{K^{4}}\right) \leq & \sum_{i<j \leq 2 K} \varrho_{\phi}\left(\left(x_{k_{i}}-x_{k_{j}}\right) 1_{A}\right) \\
= & \sum_{i<j \leq 2 K} \int_{A}\left|x_{k_{i}}-x_{k_{j}}\right| d t \\
\leq & \sum_{i<j \leq 2 K} \int_{A}\left|x_{k_{i}}-y_{i}\right|+\left|y_{i}-y_{j}\right|+\left|y_{j}-x_{k_{j}}\right| d t \\
\leq & \sum_{i<j \leq 2 K}\left(\int_{B_{i}} u_{0} d t+\int_{B_{j}} u_{0} d t+\int_{A \backslash B_{i}} \frac{d t}{K^{4} v_{0}}\right. \\
& \left.+\int_{A \backslash B_{j}} \frac{d t}{K^{4} v_{0}}+\int_{A}\left|y_{i}-y_{j}\right| d t\right)
\end{aligned}
$$




$$
\begin{aligned}
& \leq K(2 K-1) \frac{4}{K^{4}}+\int_{A} \sum_{i<j \leq 2 K}\left|y_{i}-y_{j}\right| \\
& \leq \frac{16}{K^{2}}+K^{2} u_{0} v_{0} .
\end{aligned}
$$

This implies

$$
v_{0} \geq\left(u_{0}\right)^{-1}\left(2 K^{2}-K-\frac{2}{K^{2}}-\frac{16}{K^{2}}\right) \frac{1}{K^{2}} .
$$

Since $K$ is arbitrary, $v_{0} \geq 2 / u_{0}$.

For any subsequence $\left\{x_{n_{k}}\right\}$ of $\left\{x_{n}\right\}$, define

$$
p\left(x_{n_{k}}\right)=\sup \left\{u: m\left(\left\{t: \sup \left\{x_{n_{k}}\right\}(t)-\inf \left\{x_{n_{k}}\right\}(t)>u\right\}\right)=\infty\right\} .
$$

Lemma 13. Suppose $z^{\prime}$ and $z^{\prime \prime}$ are finite almost everywhere. Then there is a subsequence $\left\{x_{n_{k}}\right\}$ of $\left\{x_{n}\right\}$ such that for any further subsequence $\left\{y_{k}\right\}$ of $\left\{x_{n_{k}}\right\}, p\left(x_{n_{k}}\right)=p\left(y_{k}\right)$.

Pr o of. For any subsequence $\left\{x_{n_{k}}\right\}$ of $\left\{x_{n}\right\}$, clearly, $p\left(x_{n}\right) \geq p\left(x_{n_{k}}\right)$. Let

$$
q\left(x_{n_{k}}\right)=\inf \left\{p\left(y_{k}\right):\left\{y_{k}\right\} \text { is a subsequence of }\left\{x_{n_{k}}\right\}\right\} \text {. }
$$

By induction, there exists a sequence $\left\{x_{j, n}: n \in \mathbb{N}\right\}_{j=1}^{\infty}$ of sequences such that

(a) for any $j,\left\{x_{j, n}: n \in \mathbb{N}\right\}$ is a subsequence of $\left\{x_{j-1, n}: n \in \mathbb{N}\right\}$;

(b) for any $j$,

$$
p_{j}=p\left(\left\{x_{j, n}: j \in \mathbb{N}\right\}\right) \leq q_{j-1}+1 / 2^{j}
$$

where $q_{j-1}=q\left(\left\{x_{j-1, n}: n \in \mathbb{N}\right\}\right)$.

Note that $\left\{p_{n}\right\}$ is a decreasing sequence, $\left\{q_{n}\right\}$ is an increasing sequence and $\left|p_{n}-q_{n-1}\right| \leq 1 / 2^{n}$. Further,

$$
u_{4}=\lim _{n \rightarrow \infty} p_{n}=\lim _{n \rightarrow \infty} q_{n}
$$

exists. We claim that $p\left(\left\{x_{n, n}: n \in \mathbb{N}\right\}\right)=u_{4}=q\left(\left\{x_{n, n}: n \in \mathbb{N}\right\}\right)$.

Let $\left\{y_{k}\right\}$ be any subsequence of $\left\{x_{n, n}: n \in \mathbb{N}\right\}$. Then for any $m \in \mathbb{N}$, $\left\{y_{k}: k \geq m\right\}$ is a subsequence of $\left\{x_{m, n}: n \in \mathbb{N}\right\}$. So

$$
p\left(y_{k}\right) \geq \lim _{m \rightarrow \infty} p\left(\left\{y_{k}: k \geq m\right\}\right) \geq \lim _{m \rightarrow \infty} q_{m}=u_{4} .
$$

For any $\varepsilon>0$, there is $m$ such that $p_{m}<u_{4}+\varepsilon / 4$. Let

$$
\begin{aligned}
& A=\left\{t: \sup \left\{x_{n, n}: n \geq m\right\}(t)-\inf \left\{x_{n, n}: n \geq m\right\}(t) \geq u_{4}+\varepsilon / 4\right\} \\
& B=\left\{t:\left|x_{j, j}(t)\right| \geq \varepsilon / 4 \text { for some } j \leq m\right\} .
\end{aligned}
$$

Since $\int_{0}^{\infty} w=\infty$ and $p\left(\left\{x_{n, n}: n \geq m\right\}\right)<u_{4}+\varepsilon / 4$, both $A$ and $B$ have finite measure. 
If $j, k \leq m$ and $t \notin A \cup B$, then

$$
\left|x_{j, j}(t)-x_{k, k}(t)\right| \leq \varepsilon / 2 \leq u_{4}+3 \varepsilon / 4
$$

$\left|\sup \left\{x_{n, n}: n \geq m\right\}(t)-x_{j, j}(t)\right|$

$$
\leq\left|\sup \left\{x_{n, n}: n \geq m\right\}(t)-x_{m, m}(t)\right|+\left|x_{m, m}(t)-x_{j, j}(t)\right| \leq u_{4}+3 \varepsilon / 4
$$

and

$$
\begin{aligned}
& \left|\inf \left\{x_{n, n}: n \geq m\right\}(t)-x_{j, j}(t)\right| \\
& \quad \leq\left|\inf \left\{x_{n, n}: n \geq m\right\}(t)-x_{m, m}(t)\right|+\left|x_{m, m}(t)-x_{j, j}(t)\right| \leq u_{4}+3 \varepsilon / 4 .
\end{aligned}
$$

This implies that for any $t \notin A \cup B, \sup \left\{x_{n, n}\right\}(t)-\inf \left\{x_{n, n}\right\}(t) \leq u_{4}+3 \varepsilon / 4$, and

$$
p\left(\left\{x_{n, n}: n \in \mathbb{N}\right\}\right) \leq u_{4}+\varepsilon .
$$

But $\varepsilon$ is arbitrary, so $p\left(\left\{x_{n, n}: n \in \mathbb{N}\right\}\right) \leq u_{4}$.

Lemma 14. Let $u_{4}, \delta$ and $\nu$ be positive real numbers. Suppose that $\left\{x_{n}\right\}$ is a unit limit-constant sequence such that for any subsequence $\left\{x_{n_{k}}\right\}$ of $\left\{x_{n}\right\}$, we have

$$
\begin{gathered}
u_{4}=p\left(\left\{x_{n}: n \in \mathbb{N}\right\}\right)=p\left(\left\{x_{n_{k}}: k \in \mathbb{N}\right\}\right), \\
m\left(\left\{t: \sup \left\{x_{n_{k}}: k \in \mathbb{N}\right\}(t)-\inf \left\{x_{n_{k}}: k \in \mathbb{N}\right\}(t)>3 \nu\right\}\right) \geq v_{0}+3 \delta .
\end{gathered}
$$

Then there is a further subsequence $\left\{x_{n_{k}}\right\}$ of $\left\{x_{n}\right\}$ such that for almost all $t$,

$$
\sup \left\{x_{n_{k}}\right\}(t)-\inf \left\{x_{n_{k}}\right\}(t) \leq u_{4} .
$$

Proof. We only prove the lemma when $v_{0}=0$. Suppose the lemma is not true. Then there is $\nu / 6>\varepsilon>0$ such that the set

$$
G_{1}=\left\{t: z^{\prime}(t)-z^{\prime \prime}(t)>u_{4}+\varepsilon\right\}
$$

has measure at least $\varepsilon$. Replace $\delta$ by $\varepsilon / 2$ if necessary. We may assume that $\delta \leq \varepsilon$. Since $v_{0}=0$, there is $0<\delta_{1}<\delta / 6$ such that if $t>\delta_{1}$, then $w(t)<w\left(\delta_{1}\right)$. Note that for any subsequence $\left\{x_{n_{k}}\right\}, p\left(x_{n_{k}}\right)=u_{4}$. Applying Lemma 8 and passing to subsequences, we may assume that for any $n \neq m$,

$$
m\left(\left\{t:\left|x_{n}(t)-x_{m}(t)\right| \geq u_{4}+2 \varepsilon / 3\right\}\right) \geq \delta_{1} .
$$

Let

$$
G_{2}=\left\{t: z^{\prime}(t)-z^{\prime \prime}(t)>u_{4}+\varepsilon / 2\right\} .
$$

Then $\delta_{1}<m\left(G_{2}\right)<\infty$, and there is $l$ such that

$$
G_{3}=\left\{t \in G_{2}: z^{\prime}(t)-z_{l}^{\prime}(t)>\varepsilon / 12 \text { and } z_{l}^{\prime \prime}(t)-z^{\prime \prime}(t)>\varepsilon / 12\right\}
$$

has measure less than $\delta_{1} / 10$. By Lemma 7 , there is $N_{3}$ such that for any $n>N_{3}$, the set

$$
G_{4}=\left\{t \in G_{2} \backslash G_{3}: \text { either }\left|z_{l}^{\prime}(t)-x_{n}(t)\right|<\varepsilon / 6 \text { or }\left|z_{l}^{\prime \prime}(t)-x_{n}(t)\right|<\varepsilon / 6\right\}
$$


has measure at least $m\left(G_{2}\right)-\delta_{1} / 5$. Let

$$
\begin{aligned}
G_{5}=G_{4} \cap G_{1}=\left\{t \in G_{1} \backslash G_{3}\right. & : \text { either }\left|z_{l}^{\prime}(t)-x_{n}(t)\right|<\varepsilon / 6 \\
& \text { or } \left.\left|z_{l}^{\prime \prime}(t)-x_{n}(t)\right|<\varepsilon / 6\right\} .
\end{aligned}
$$

Then $m\left(G_{5}\right) \geq m\left(G_{1}\right)-\delta_{1} / 5$ and for any $t \in G_{5}$ (respectively, $t \in G_{4}$ ), there exists $k_{1} \leq l$ (respectively, $k_{2} \leq l$ ) such that

$$
\left|x_{n}(t)-x_{k_{1}}(t)\right|=\max \left\{\left|x_{n}(t)-z_{l}^{\prime}(t)\right|,\left|x_{n}(t)-z_{l}^{\prime \prime}(t)\right|\right\} \geq u_{4}+\varepsilon-\varepsilon / 3,
$$

or respectively,

$$
\left.\left|x_{n}(t)-x_{k_{2}}(t)\right|=\min \left\{\left|x_{n}(t)-z_{l}^{\prime}(t)\right|,\left|x_{n}(t)-z_{l}^{\prime \prime}(t)\right|\right\} \leq \varepsilon / 6\right) .
$$

Since $\left\{x_{n}\right\}$ is a unit limit-constant sequence, for any $\lambda>0$, there are $n>N_{3}$ and a measure preserving transformation $\sigma$ such that

(i) $\int_{0}^{\infty} \phi\left(x_{n}-\bar{x}_{l}\right) w \circ \sigma=\int_{0}^{\infty} \phi\left(\left(x_{n}-\bar{x}_{l}\right)^{*}\right) w \geq 1-\lambda$;

(ii) if $\left|\left(x_{n}-\bar{x}_{l}\right)(t)\right| \geq\left|\left(x_{n}-\bar{x}_{l}\right)(s)\right|$, then $\sigma(t) \geq \sigma(s)$.

Case 1: $m\left(\sigma^{-1}\left(0, \delta_{1}\right) \cap G_{4}\right) \geq 2 \delta_{1} / 5$. For any $k \leq l$, let

$$
H_{k}=\left\{t: t \in \sigma^{-1}\left(0, \delta_{1}\right) \text { and }\left|x_{n}(t)-x_{k}(t)\right| \leq \varepsilon / 6\right\} .
$$

Since $\bigcup_{k=1}^{l} H_{k} \supseteq G_{4} \cap \sigma^{-1}\left(0, \delta_{1}\right)$, there exists $k \leq l$ such that $m\left(H_{k}\right) \geq$ $2 \delta_{1} /(5 l)$. By $(4)$,

$$
m\left(\left\{t \in \sigma^{-1}\left(\delta_{1}, \infty\right):\left|x_{n}(t)-x_{k}(t)\right| \geq u_{4}+2 \varepsilon / 3\right\}\right) \geq 2 \delta_{1} /(5 l) .
$$

Case 2: $\left.m\left(\sigma^{-1}\left(0, \delta_{1}\right)\right) \cap G_{4}\right)<2 \delta_{1} / 5$. Note that $G_{5} \subseteq G_{4} \subseteq G_{2}$ and $m\left(G_{2}\right) \leq \delta_{1} / 5+m\left(G_{4}\right)$. We have

$$
m\left(\sigma^{-1}\left(0, \delta_{1}\right) \backslash G_{2}\right) \geq \delta_{1}-m\left(\sigma^{-1}\left(0, \delta_{1}\right) \cap G_{4}\right)-\delta_{1} / 5 \geq 2 \delta_{1} / 5,
$$

and

$$
\begin{aligned}
4 \delta_{1} / 5 & \leq m\left(G_{1}\right)-\delta_{1} / 5 \\
& \leq m\left(G_{5}\right)=m\left(\sigma^{-1}\left(\delta_{1}, \infty\right) \cap G_{5}\right)+m\left(\sigma^{-1}\left(0, \delta_{1}\right) \cap G_{5}\right) \\
& \leq m\left(\sigma^{-1}\left(\delta_{1}, \infty\right) \cap G_{5}\right)+m\left(\sigma^{-1}\left(0, \delta_{1}\right) \cap G_{4}\right) \\
& \leq m\left(\sigma^{-1}\left(\delta_{1}, \infty\right) \cap G_{5}\right)+2 \delta_{1} / 5 .
\end{aligned}
$$

This yields

$$
m\left(\sigma^{-1}\left(\delta_{1}, \infty\right) \cap G_{5}\right) \geq 4 \delta_{1} / 5-2 \delta_{1} / 5=2 \delta_{1} / 5 .
$$

Let

$$
H_{k}^{\prime}=\left\{t \in \sigma^{-1}\left(\delta_{1}, \infty\right):\left|x_{n}(t)-x_{k}(t)\right| \geq u_{4}+2 \varepsilon / 3\right\} .
$$

Let $t$ be an element of $G_{5} \cap \sigma^{-1}\left(\delta_{1}, \infty\right)$. Then

$$
z^{\prime}(t)-z^{\prime \prime}(t)>u_{4}+\varepsilon
$$

with either $\left|z_{l}^{\prime}(t)-x_{n}(t)\right|<\varepsilon / 6$ or $\left|z_{l}^{\prime \prime}(t)-x_{n}(t)\right|<\varepsilon / 6$. So $t \in H_{k}^{\prime}$ for some $k \leq l$. By (5), there is $k \leq l$ such that $m\left(H_{k}^{\prime}\right) \geq \delta_{1} /(5 l)$. On the other hand, if $t \in \sigma^{-1}\left(0, \delta_{1}\right) \backslash G_{2}$, then $\left|x_{n}(t)-x_{k}(t)\right| \leq z^{\prime}(t)-z^{\prime \prime}(t) \leq u_{4}+\varepsilon / 2$. 
By Lemma 2 and Remark 1, for both cases, there is $\delta_{2}>0$ (which is dependent only on $\left.\delta_{1}, l, u_{4}, \varepsilon\right)$ such that

$$
\varrho_{\phi}\left(x_{n}-x_{k}\right) \geq \int \phi\left(x_{n}-x_{k}\right) w \circ \sigma+\delta_{2} .
$$

This implies, for any $\lambda>0$,

$$
\begin{aligned}
1-\lambda & \leq \int \phi\left(x_{n}-\frac{1}{l} \sum_{j=1}^{l} x_{j}\right) w \circ \sigma \leq \frac{1}{l} \sum_{j=1}^{l} \int \phi\left(x_{n}-x_{j}\right) w \circ \sigma \\
& \leq \frac{1}{l} \sum_{j=1}^{l} \varrho_{\phi}\left(x_{n}-x_{j}\right)-\frac{\delta_{2}}{l} \leq 1-\frac{\delta_{2}}{l} .
\end{aligned}
$$

This is impossible if $\lambda<\delta_{2} / l$.

3. Proof of Theorem 1. Let $\Lambda_{\phi, w}$ be an order continuous LorentzOrlicz space such that $\int_{0}^{v_{0}} \phi\left(u_{0}\right) w<2$. We claim that if $\Lambda_{\phi, w}$ contains a unit limit-constant sequence $\left\{x_{n}\right\}$, then

(a) $\left\{x_{n}\right\}$ does not converge weakly;

(b) if $\Lambda_{\phi, w} \equiv \Lambda_{\phi, w}(0,1)$, then $u_{0}=\infty$.

Condition (a) implies that if $\int_{0}^{v_{0}} \phi\left(u_{0}\right) w<2$, then $\Lambda_{\phi, w}$ has weakly normal structure. By Lemma 6 (cf. Remark 2), (b) yields that $u_{0}>0$ if $\Lambda_{\phi, w}$ does not have normal structure. Moreover, if $\Lambda_{\phi, w} \equiv \Lambda_{\phi, w}(0,1)$ does not have normal structure, then either $\int_{0}^{v_{0}} \phi\left(u_{0}\right) w \geq 2$ or $u_{0}=\infty$.

Let $\left\{x_{n}\right\}$ be a unit limit-constant sequence in $\Lambda_{\phi, w}$. Suppose that $\left\{x_{n}\right\}$ satisfies one of the following conditions:

(c) For any $M>0$, there is $n$ such that $\varrho_{\phi}\left(x_{n} 1_{\left\{t:\left|x_{n}(t)\right|>M\right\}}\right)>\delta$.

(d) For any $\varepsilon>0$ there is $n$ such that $\varrho_{\phi}\left(x_{n} 1_{\left\{t:\left|x_{n}(t)\right|<\varepsilon\right\}}\right)>\delta$.

By Proposition 11, $\left\{x_{n}\right\}$ does not contain any weakly covergent subsequence. By Lemma 6, (c) yields $u_{0}=\infty$.

Suppose (d) holds. Since $\Lambda_{\phi, w}$ is order continuous, for any $\delta>0$ there is $\varepsilon>0$ such that $\varrho_{\phi}\left(\varepsilon 1_{(0,1)}\right)<\delta / 2$. Hence, if $\varrho_{\phi}\left(x_{n} 1_{\left\{t:\left|x_{n}(t)\right|<\varepsilon\right\}}\right)>\delta$, then we must have $\Lambda_{\phi, w} \equiv \Lambda_{\phi, w}(0, \infty)$. Hence we may assume that neither (c) nor (d) holds.

Since $\int_{0}^{v_{0}} \phi\left(u_{0}\right) w<2$, by Proposition 12, there exists $\nu>0$ such that for any subsequence $\left\{x_{n_{k}}\right\}$ of $\left\{x_{n}\right\}$,

$$
m\left(\left\{t: \sup \left\{x_{n_{k}}: k \in \mathbb{N}\right\}(t)-\inf \left\{x_{n_{k}}: k \in \mathbb{N}\right\}(t)>3 \nu\right\}\right) \geq v_{0}+3 \nu .
$$

The same assumption yields either $u_{0}<\infty$ or $v_{0}=0$. By Lemma 6 and Corollary 9 , both $z^{\prime}$ and $z^{\prime \prime}$ are finite almost everywhere. Applying Lemmas 13 and 14 and passing to further subsequences of $\left\{x_{n}\right\}$, we may assume 
that for any subsequence $\left\{x_{n_{k}}\right\}$ of $\left\{x_{n}\right\}$,

$$
u_{4}=p\left(x_{n}\right)=p\left(x_{n_{k}}\right),
$$

and

$$
\sup \left\{x_{n}\right\}(t)-\inf \left\{x_{n}\right\}(t) \leq u_{4} .
$$

If $u_{4}=0$, then $\left\{x_{n}\right\}$ contains a constant subsequence. This contradicts the fact that $\left\{x_{n}\right\}$ is a unit limit-constant sequence. So $u_{4}$ must be positive,

$$
m\left(\left\{t: \sup \left\{x_{n}\right\}(t)-\inf \left\{x_{n}\right\}(t)>15 u_{4} / 16\right\}\right)=\infty,
$$

and $\Omega=(0, \infty)$. By Corollary 10 , there is $v$ such that $w$ is constant on $(v, \infty)$. Let

$$
v_{1}=\inf \{v: w \text { is constant on }(v, \infty)\} .
$$

If $v_{1}=0$, then $v_{0}=\infty$. This contradicts our assumption $\int_{0}^{v_{0}} \phi\left(u_{0}\right) w<2$. So $v_{1} \geq v_{0}$ and $v_{1}>0$.

By (8) and Lemma 8, there exists a subsequence $\left\{x_{n_{k}}\right\}$ of $\left\{x_{n}\right\}$ such that for any $j<m$,

$$
\left.m\left(\left\{t:\left|x_{n_{j}}-x_{n_{m}}\right| \geq 7 u_{4} / 8\right)\right\}\right) \geq v_{1} .
$$

Replacing $\left\{x_{k}\right\}$ by $\left\{x_{n_{k}}\right\}$ if necessary, we may assume that for any $n>m$,

$$
\left.m\left(\left\{t:\left|x_{n}(t)-x_{m}(t)\right| \geq 7 u_{4} / 8\right)\right\}\right) \geq v_{1} .
$$

Claim. There are a subsequence $\left\{x_{n_{k}}\right\}$ of $\left\{x_{n}\right\}$ and a sequence of pairwise disjoint measurable sets $\left\{B_{k}\right\}$ such that $m\left(B_{k}\right) \geq 2 v_{1} / 3$, and for any $m \in \mathbb{N}, t \in B_{m}$,

$$
\left|x_{n_{m}}(t)-x_{n_{k}}(t)\right| \geq 3 u_{4} / 4 \quad \text { if } k<m .
$$

Suppose the claim were proved. By the proof of Example $3,\left\{\left(x_{n_{k}}-\right.\right.$ $\left.\left.x_{n_{k-1}}\right) 1_{B_{k}}\right\}$ is equivalent to the natural basis of $\ell_{1}$. By Proposition $5,\left\{x_{n}\right\}$ does not converge weakly. Hence we only need to prove our claim.

Proof of Claim. Let $n_{1}=2$. Suppose that $n_{1}, \ldots, n_{k}$ are selected. For any $l>n_{k}$ with $\varrho_{\phi}\left(x_{l}-(1 / k) \sum_{j=1}^{k} x_{n_{j}}\right)>1-\lambda$, let $\sigma$ be the measure preserving transformation such that $1-\lambda$

(i) $\int_{0}^{\infty} \phi\left(x_{l}-(1 / k) \sum_{j=1}^{k} x_{n_{j}}\right) w \circ \sigma=\int_{0}^{\infty} \phi\left(\left(x_{l}-(1 / k) \sum_{j=1}^{k} x_{n_{j}}\right)^{*}\right) w \geq$ $\sigma(s)$.

(ii) if $\left.\left|\left(x_{l}-(1 / k) \sum_{j=1}^{k} x_{n_{j}}\right)(t)\right| \geq \mid x_{l}-(1 / k) \sum_{j=1}^{k} x_{n_{j}}\right)(s) \mid$, then $\sigma(t) \geq$

If $m\left(\left\{t \in \sigma^{-1}\left(\left(0, v_{1}\right)\right):\left|x_{l}(t)-x_{n_{j}}(t)\right| \leq 3 u_{4} / 4\right\}\right) \geq v_{1} / 4^{j}$ for some $j \leq k$, then by (9), we have

$$
m\left(\left\{t \in \sigma^{-1}\left(v_{1}, \infty\right):\left|x_{l}(t)-x_{n_{j}}(t)\right| \geq 7 u_{4} / 8\right\}\right) \geq v_{1} / 4^{j} .
$$


By Lemma 2 and Remark 1, there is $\delta_{3}>0$ independent of $\sigma$ such that

$$
\int_{0}^{\infty} \phi\left(x_{l}-\frac{1}{k} \sum_{j=1}^{k} x_{n_{j}}\right) w \circ \sigma \leq 1-\delta_{3} .
$$

Since $\Lambda_{\phi, w}$ is order continuous and $\left\{x_{n}\right\}$ is a unit limit-constant sequence, there is $n_{k+1}>n_{k}$ such that

$$
\int_{0}^{\infty} \phi\left(x_{l}-\frac{1}{k} \sum_{j=1}^{k} x_{n_{j}}\right) w \circ \sigma \geq 1-\frac{\delta_{3}}{2} .
$$

The above proof shows that for any $j \leq k$,

Let

$$
m\left(\left\{t \in \sigma^{-1}\left(0, v_{1}\right):\left|x_{n_{k+1}}(t)-x_{n_{j}}(t)\right| \leq 3 u_{4} / 4\right\}\right) \leq v_{1} / 4^{j} .
$$

$$
B_{k+1}=\left\{t \in \sigma^{-1}\left(0, v_{1}\right): \text { for any } j \leq k,\left|x_{l}(t)-x_{n_{j}}(t)\right|>3 u_{4} / 4\right\} .
$$

Then

$$
m\left(B_{k+1}\right) \geq v_{1}-\sum_{j=1}^{k} \frac{v_{1}}{4^{j}} \geq \frac{2 v_{1}}{3} .
$$

Let $t$ be an element in $B_{k}$ and $i, j$ two natural numbers such that $i<j<k$. Then

$$
(1+1 / 16) u_{4} \geq\left|x_{n_{k}}(t)-x_{n_{j}}(t)\right|>3 u_{4} / 4 \quad \text { by }(7) .
$$

If $\left(x_{n_{k}}(t)-x_{n_{j}}(t)\right)\left(x_{n_{k}}(t)-x_{n_{i}}(t)\right)<0$, then

$$
\begin{aligned}
\left|x_{n_{i}}(t)-x_{n_{j}}(t)\right| & =\left|x_{n_{k}}(t)-x_{n_{j}}(t)\right|+\left|x_{n_{k}}(t)-x_{n_{i}}(t)\right| \\
& \geq 2 \frac{3 u_{4}}{4}=\frac{3 u_{4}}{2} .
\end{aligned}
$$

This is impossible. So for almost all $t \in B_{k+1}$ and for $i<j<k, \operatorname{sgn}\left(x_{n_{k}}(t)-\right.$ $\left.x_{n_{j}}(t)\right)=\operatorname{sgn}\left(x_{n_{k}}(t)-x_{n_{i}}(t)\right)$, and

$$
\left|x_{n_{i}}(t)-x_{n_{j}}(t)\right| \leq u_{4} / 4 .
$$

This implies $t \notin B_{j}$ and $\left\{B_{k}\right\}$ is pairwise disjoint. We proved our claim, and hence also Theorem 1.

Re mark 3. (1) The results in Sections 2 and 3 are still true for LorentzOrlicz sequence spaces $\ell_{\phi, w}$. Hence if $\ell_{\phi, w}$ is an order continuous LorentzOrlicz sequence space (i.e. $\phi$ satisfies the $\Delta_{2}$ condition for small values and $\left.\sum_{i=1}^{\infty} w(i)=\infty\right)$, then $\ell_{\phi, w}$ has normal structure if and only if $u_{0}=0$.

(2) Let $\left\{x_{n}\right\}$ be a limit-constant sequence in an order continuous LorentzOrlicz sequence space $\ell_{\phi, w}$. We claim that $\left\{x_{n}\right\}$ does not converge weakly. By passing to a subsequence and then translating it, we may assume that for any $n>m$,

$$
\left\|\left|x_{n}\right| \wedge\left|x_{m}\right|\right\|_{\infty} \leq 1 / n
$$


If for any $\varepsilon>0$ there is $n \in \mathbb{N}$ such that $\left\|x_{n}\right\|_{\infty}<\varepsilon$, then by Proposition 11, $\left\{x_{n}\right\}$ does not converge weakly. In this case, we are done. So we may assume that there is $N$ and $\varepsilon>0$ such that $\left\|x_{n}\right\|_{\infty} \geq \varepsilon$ for all $n>N$. By Corollary 10 , there is $v \geq 0$ such that $w$ is constant on $(v, \infty)$. By Proposition 5 (cf. Example 3), $\left\{x_{n}\right\}$ does not converge weakly. Hence every order continuous Lorentz-Orlicz sequence space $\ell_{\phi, w}$ has weakly normal structure.

\section{References}

[1] N. L. Carothers, S. J. Dilworth, C. J. Lennard and D. A. Trautman, A fixed point property for the Lorentz space $L_{p, 1}(\mu)$, Indiana Univ. Math. J. 40 (1991), $345-352$.

[2] N. L. Carothers, R. Haydon and P.-K. Lin, On the isometries of the Lorentz function spaces, Israel J. Math. 84 (1993), 265-287.

[3] S. Chen, Geometry of Orlicz spaces, Dissertationes Math. 356 (1996).

[4] J. Diestel, Sequences and Series in Banach Spaces, Springer, 1984.

[5] S. J. Dilworth and Y.-P. Hsu, The uniform Kadec-Klee property for the Lorentz space $L_{w, 1}$, J. Austral. Math. Soc. Ser. A 60 (1996), 7-17.

[6] D. V. van Dulst and V. D. de Valk, (KK)-properties, normal structure and fixed points of nonexpansive mappings in Orlicz sequence spaces, Canad. J. Math. 38 (1986), 728-750.

[7] A. Kamińska, Some remarks on Orlicz-Lorentz spaces, Math. Nachr. 147 (1990), $29-38$.

[8] A. Kamińska, P.-K. Lin and H. Y. Sun, Uniformly normal structure of OrliczLorentz spaces, in: Interaction between Functional Analysis, Harmonic Analysis, and Probability, N. Kalton, E. Saab and S. Montgomery-Smith (eds.), Lecture Notes in Pure and Appl. Math. 175, Dekker, New York, 1996, 229-238.

[9] W. A. Kirk, A fixed point theorem for mappings which do not increase distances, Amer. Math. Monthly 72 (1965), 1004-1006.

[10] T. Landes, Permanence properties of normal structure, Pacific J. Math. 110 (1984), $125-143$.

[11] - Normal structure and weakly normal structure of Orlicz sequence spaces, Trans. Amer. Math. Soc. 285 (1984), 523-534.

[12] P.-K. Lin and H. Y.S un, Some geometric properties of Lorentz-Orlicz spaces, Arch. Math. (Basel) 64 (1995), 500-511.

[13] J. Lindenstrauss and L. Tzafriri, Classical Banach Spaces II, Springer, 1979.

[14] M. M. Rao and Z. D. Ren, Theory of Orlicz Spaces, Marcel Dekker, 1991.

Department of Mathematics

University of Memphis

Memphis, Tennessee 38152, U.S.A.

E-mail: linpk@hermes.msci.memphis.edu
Department of Mathematics Harbin Institute of Technology Harbin, China 